OPEN ACCESS

Edited by:

Ding She,

Tsinghua University, China

Reviewed by:

Qian Zhang,

Harbin Engineering University, China

Guang $\mathrm{Hu}$,

X'an Jiaotong University, China

*Correspondence:

Deokjung Lee

deokjung@unist.ac.kr

Specialty section:

This article was submitted to

Nuclear Energy,

a section of the journal

Frontiers in Energy Research

Received: 27 August 2021 Accepted: 08 November 2021 Published: 20 December 2021

Citation:

Choi S, Kim W and Lee D (2021)

Refinements of the Pin-Based

Pointwise Energy Slowing-Down Method for Resonance Self-Shielding

Calculation-l: Theory.

Front. Energy Res. 9:765863.

doi: 10.3389/fenrg.2021.765863

\section{Refinements of the Pin-Based Pointwise Energy Slowing-Down Method for Resonance Self-Shielding Calculation-I: Theory}

\author{
Sooyoung Choi ${ }^{1}$, Wonkyeong Kim $^{2}$ and Deokjung Lee ${ }^{2 *}$ \\ ${ }^{1}$ Department of Nuclear Engineering and Radiological Sciences, University of Michigan, Ann Arbor, MI, United States, \\ ${ }^{2}$ Department of Nuclear Engineering, Ulsan National Institute of Science and Technology, Ulsan, South Korea
}

The pin-based pointwise energy slowing-down method (PSM), which is a resonance selfshielding method, has been refined to treat the nonuniformity of material compositions and temperature profile in the fuel pellet by calculating the exact collision probability in the radially subdivided fuel pellet under the isolated system. The PSM has generated the collision probability table before solving the pointwise energy slowing-down equation. It is not exact if the fuel pellet has nonuniform material compositions or temperature profile in all the subdivided regions. In the refined PSM-CPM, the pre-generated table is not required for directly calculating the collision probability in all the subdivided regions of the fuel pellet while solving the slowing-down equation. There are an advantage and a disadvantage to the method. The advantage is to exactly consider the nonuniformity of the material compositions and temperature profile in the fuel pellet. The disadvantage is the longer computing time than that of the PSM when the fuel pellet has more than five subdivided regions. However, in the practical use for $\mathrm{UO}_{2}$ pin-cells, it is still comparable for the computation time with the PSM and the conventional equivalence theory methods. In this article, using simple light water reactor $17 \times 17 \mathrm{FA}$ problems with a uniform material composition and temperature profile, it is demonstrated that PSMs (PSM and PSM-CPM) exhibit consistent accuracy in calculating the multiplication factor and the pin power distribution with no compromise in the computation time. More detailed accuracy assessments with various test cases, including problems representing the nonuniformity, are presented in the accompanying article.

Keywords: resonance self-shielding calculation, equivalence theory, pointwise energy slowing-down method, resonance treatment, light water reactor, reactor physics

\section{INTRODUCTION}

In reactor physics, all calculations are conducted with cross sections (XSs). The actual XSs for nuclides describe the very detailed energy resolution. XS data are composed of hundreds of thousands of energy points for major resonant nuclides (e.g. $\left.{ }^{238} \mathrm{U}\right)$. Even with modern computing resources, it is still time consuming and impractical to directly utilize raw XS data in lattice physics calculations. Because of this, XSs, especially in the resonance energy range that requires high-energy resolution, must be condensed during neutron transport computations. A resonance self-shielding calculation (or resonance treatment) is performed to condense a detailed XS into the multigroup level (e.g., $\sim 100$ groups). 
Therefore, if the resonance self-shielding calculation is not accurate, all the subsequent calculations would not be meaningful. The resonance treatment is one of the most difficult and challenging parts of reactor physics.

The equivalence theory has been widely used for resonance treatment (Knott et al., 2010; Stamm'ler and Abbate, 1983). The equivalence theory gives a reasonable solution within a short computation time. Owing to this advantage, many lattice physics codes adopt the equivalence theory to generate effective multigroup XSs (Powney and Hutton, 2002; Rhodes et al., 2006). There has been much research into the equivalence theory to improve the accuracy of effective multigroup XSs and the applicability to general geometry (Koike et al., 2012; Godfrey, 2014; Choi et al., 2015a). However, there are still many points missing from the resonance calculation. The author's previous works have determined that resonance scattering causes the overestimation of the ${ }^{238} \mathrm{U}$ effective XS (Choi, 2017; Choi et al., 2017).

In one of the authors' previous work (Choi et al., 2017), a new resonance self-shielding method, the pin-based pointwise slowing-down method (PSM), was developed to resolve limitations, that is, the overestimation of ${ }^{238} \mathrm{U}$ XS due to resonance scattering sources, in the conventional equivalence theory. However, the PSM has an assumption for treating with the total XSs in the subdivided regions of the fuel pellet. As one of the techniques for achieving high performance, the PSM generates the collision probability of the isolated fuel pellet before solving the pointwise slowing-down equation. The collision probability is generated with the grid of the total XSs that is assumed to be constant in the subdivided regions of the fuel pellet. With the nonuniform material and temperature distribution, the total XSs in the subdivided regions are different from each other so that the accuracy of the effective XSs is impeded by the constant total XS assumption (Zhang et al., 2018; Zhang et al., 2020). Therefore, generating the collision probability during solving the slowing-down equation on the fly, a more rigorous method, PSM-CPM, has been developed to treat nonuniform material and temperature distributions in the fuel pellet.

This work reviews the PSM in brief and introduces PSM-CPM with the refinement. The work demonstrates the accuracy of the PSM and PSM-CPM with several light water reactor problems with only uniform material and temperature distributions. The computing time for the two methods is also estimated and compared. Detailed verifications of the PSM-CPM are performed with various light water reactor (LWR) problems with a nonuniform material composition and temperature profile, and the detailed XS comparison is presented to show the superior accuracy of the method in the accompanying article (Kim et al., 2021).

\section{PIN-BASED POINTWISE ENERGY SLOWING-DOWN METHODS (PSMS)}

The achievements of the PSM are summarized as follows: RI or XS look-up tables are not required for resonance treatment; the distribution of the scattering sources in the fuel pellet is accurately modeled; and PSMs have a comparable computational cost with the equivalence theory.

The neutron transport equation can be expressed with the radially subdivided regions (Stoker and Weiss, 1996) and collision probabilities for the two-region problem (i.e., fuel and moderator) in the resonance energy range as follows:

$$
\Sigma_{t, i}(E) \phi_{i}(E) V_{i}=\sum_{j \in F} P_{j i}(E) V_{j} Q_{s, j}(E)+P_{M i}(E) V_{M} Q_{s, M}(E),
$$

where $i$ and $j$ are the indexes of the radially subdivided regions of the fuel pellet, $\Sigma_{t, i}(E)$ is the total XS of the radially subdivided region $i$ of the fuel pellet, $\phi_{i}(E)$ is the flux in the subdivided region $i, V_{i}$ is the volume of the subdivided region $i, F$ is the index of the fuel pellet, $M$ is the index of the moderator, $P_{j i}(E)$ is the collision probability from the subdivided region $j$ to the subdivided region $i$, and the slowing-down scattering source of the subdivided region $j$ is defined as follows:

$$
Q_{s, j}(E)=\sum_{r \in j} N^{r} \int_{E}^{E / \alpha^{r}} \sigma_{s}^{r}\left(E^{\prime}\right) \phi_{j}\left(E^{\prime}\right) \frac{1}{1-\alpha^{r}} \frac{d E^{\prime}}{E^{\prime}} .
$$

Using the lethargy form and the reciprocity relation, Eq. $\mathbf{1}$ is written as follows:

$$
\begin{aligned}
\sum_{t, i}(u) \phi_{i}(u)= & \sum_{j \in F} P_{i j}(u) \frac{\sum_{t, i}(u)}{\sum_{t, j}(u)} Q_{s, j}(u) \\
& +P_{i M}(u) \frac{\sum_{t, i}(u)}{\sum_{p, M}} Q_{s, M}(u), \quad i \in F .
\end{aligned}
$$

The flux of the subdivided region $i$ by rearranging Eq. 3 is expressed as follows:

$$
\phi_{i}(u)=\sum_{j \in F} \frac{P_{i j}(u)}{\sum_{t, j}(u)} Q_{s, j}(u)+\frac{P_{i M}(u)}{\sum_{p, M}} Q_{s, M}(u), \quad i \in F .
$$

In Eq. 3, the index $M$ also indicates the nonfuel region. It is assumed that materials in the nonfuel region only have a potential $\mathrm{XS}$. In case of the multiregion problem, the nonfuel regions (i.e., $\mathrm{gap} / \mathrm{clad} /$ moderator) in a unit pin-cell are merged into a single region with the volume weighting by assuming constant spatial fluxes in the nonfuel regions.

The transport equation for the nonfuel region is written as follows:

$$
\Sigma_{p, M} \phi_{M}(u) V_{M}=\sum_{i \in F} P_{i M}(u) V_{i} Q_{s, i}(u)+P_{M M}(u) V_{M} Q_{s, M}(u),
$$

where $P_{M M}(u)$ is the nonfuel-to-nonfuel collision probability.

The flux of the nonfuel region $M$ by rearranging Eq. 5 is as follows:

$$
\phi_{M}(u)=\sum_{i \in F} \frac{P_{M i}(u)}{\sum_{t, i}(u)} Q_{s, i}(u)+\frac{P_{M M}(u)}{\sum_{p, M}} Q_{s, M}(u) .
$$

If $P_{i j}(u), P_{i M}(u), P_{M i}(u)$, and $P_{M M}(u)$ are known, the fluxes and scattering sources in Eqs 4-6 can be calculated by solving a fixed source transport equation. 
The PSM and PSM-CPM calculate the collision probability with a two-step approach. In the first step, the collision probabilities of the subdivided regions in the isolated fuel pellet are calculated.

The difference between PSM and PSM-CPM is the way to calculate $P_{i j}^{i s o}$, whereby a neutron uniformly born in the subdivided region $i$ has its first collision in the subdivided region $j$. The PSM-CPM calculates $P_{i j}^{i s o}$ by using the CPM (Carlvik, 1996) for all energy points while solving the slowingdown equations with pointwise XSs.

In the PSM, however, $P_{i j}^{i s o}$ is tabulated as a function of the total $\mathrm{XS}$ of the fuel pellet before solving the slowing-down equations, and then $P_{i j}^{i s o}$ is interpolated using the total XS of any energy of interest. While solving the slowing-down equations, $P_{i j}^{i s o}$ is interpolated from the $\hat{P}_{i j}^{i s o}$ table using the total XS of the fuel pellet at any energy of interest as follows:

$$
P_{i j}^{i s o}(u)=\hat{P}_{i j}^{i s o}\left(\Sigma_{t, F}(u)\right) .
$$

Equation 7 is only exact if the fuel pellet has a constant material composition and temperature profile in all the subdivided regions. However, if the fuel is burned, the material compositions in the subdivided regions change differently from each other. For the burned fuel pellet, the burnup of the outermost subregion is higher than that in the inner subregion because of the spatial self-shielding effect. In addition, the thermal-hydraulic (TH) calculation is coupled with neutronics to analyze the power reactor. The fuel pellet must be divided into several rings to model the temperature profile from TH feedback. With the TH feedback, the temperature in the inner region is higher than that in the outer region. Obviously, the pointwise energy XSs depend on the material composition and the temperature. With nonuniform material compositions and temperatures, the total XSs of the subdivided regions are different from each other. In this case, an average total XS (i.e., $\left.\bar{\Sigma}_{t, F}\right)$ is defined with Eq. 8, and the total XS of the entire fuel pellet (i.e., $\Sigma_{t, F}$ ) in Eq. 7 is replaced by the average total XS.

$$
\Sigma_{t, F}(u) \approx \bar{\Sigma}_{t, F}(u)=\frac{\sum_{i \in F} \Sigma_{t, i}(u) \phi_{i}\left(u^{h}\right) V_{i}}{\sum_{i \in F} \phi_{i}\left(u^{h}\right) V_{i}},
$$

where energy $u^{h}$ is one point higher than energy $u$.

Introducing the $\hat{P}_{i j}^{i s o}$ table can lead error in computing the collision probability, even though it can significantly reduce the calculation time. The accuracy and efficiency on the use of the pre-generated table are estimated and compared in Section 3 and the accompanying article (Kim et al., 2021) in detail.

$P_{i j}^{i s o}$ considers the collision probability of an isolated fuel rod. Therefore, a proper correction is required to consider the shadowing effect from neighboring fuel rods and structural materials. In this second step of the collision probability calculation, it is assumed that the shadowing effect is not significantly different for the individually subdivided regions of the fuel pellet. In other words, the subdivided regions of the fuel pellet have the same shadowing effect. Under this assumption, a multiterm rational equation in the equivalence theory is used.
In PSMs, the shadowing correction factor is calculated with two escape probabilities. One is the fuel escape probability of an isolated fuel pin, and the other is that of the fuel pin in the lattice (or core). Carlvik's two-term rational approximation (Knott et al., 2010) is applied for a cylindrical geometry to calculate $P_{e, F}^{i s o}(u)$ and $P_{e, F}(u)$ in Eqs 22, 23. $P_{e, F}^{i s o}$ is the fuel escape probability of the isolated fuel pellet, and $P_{e, F}$ is the fuel escape probability of the fuel pellet in the lattice.

$$
\begin{gathered}
P_{e, F}^{i s o}(u)=1-P_{F F}^{i s o}(u)=2 \frac{2 \Sigma_{e, F}}{\sum_{t, F}(u)+2 \Sigma_{e, F}}-\frac{3 \Sigma_{e, F}}{\Sigma_{t, F}(u)+3 \Sigma_{e, F}} ; \quad(9) \\
P_{e, F}(u)=1-P_{F F}(u)=\beta_{1} \frac{\alpha_{1} \Sigma_{e, F}}{\sum_{t, F}(u)+\alpha_{1} \Sigma_{e, F}}+\beta_{2} \frac{\alpha_{2} \Sigma_{e, F}}{\sum_{t, F}(u)+\alpha_{2} \Sigma_{e, F}},
\end{gathered}
$$

where $P_{e, F}^{i s o}$ is the fuel escape probability of the isolated fuel pellet, $P_{e, F}$ is the fuel escape probability of the fuel pellet in lattice, and the coefficients in Eq. $\mathbf{1 0}$ are defined with the Dancoff factor as follows:

$$
\left\{\begin{array}{l}
A=\Gamma /(1-\Gamma) \\
\alpha_{1,2}=\left(5 A+6 \mp \sqrt{A^{2}+36 A+36}\right) /(2 A+2) \\
\beta_{1}=\left(\frac{4 A+6}{A+1}-\alpha_{1}\right) /\left(\alpha_{2}-\alpha_{1}\right) \\
\beta_{2}=1-\beta_{1}
\end{array}\right.
$$

where $\Gamma$ is the Dancoff factor of the fuel pellet.

It should be noted that $P_{e, F}^{i s o}$ and $P_{e, F}$ are probabilities for the fuel pellet, not individual subregions of the pellet. The total XS of the fuel pellet, $\Sigma_{t, F}$, to calculate both escape probabilities is calculated by taking the average of the total XSs of the subdivided regions of the fuel pellet. Although Eq. 8 is only used for the PSM, the average XS of the fuel pellet is needed for both PSM and PSM-CPM to consider the shadowing effect.

The shadowing effect correction factor, which adjusts the fuel escape probability of an isolated fuel pin to consider the shadowing effect, is defined as a ratio of the fuel escape probabilities of two systems in the isolated fuel pellet and the lattice as follows:

$$
\eta_{F}(u)=\frac{P_{e, F}(u)}{P_{e, F}^{i s o}(u)} .
$$

The shadowing effect correction factor is multiplied by the fuel escape probability in each subregion of the fuel pellets as follows:

$$
P_{i M}(u)=P_{e, i}(u)=\eta_{i}(u) P_{e, i}^{i s o}(u),
$$

where

$$
\begin{gathered}
P_{i M}^{i s o}(u)=P_{e, i}^{i s o}(u)=1-\sum_{j \in F} P_{i j}^{i s o}(u) \\
\eta_{i}(u) \approx \eta_{F}(u) .
\end{gathered}
$$

It is assumed that the shadowing effect correction factor of the subdivided region $i$ is equal to that of the fuel pellet, as shown in Eq. 15. The source distribution in the fuel pellet is very important because it has a significant effect on the fuel escape probability. When $P_{e, F}$ and $P_{e, F}^{i s o}$ are calculated, a constant source distribution 
in the fuel pellet is assumed. Therefore, the probabilities are not exact. The error from the constant source assumption exists in both $P_{e, F}$ and $P_{e, F}^{i s o}$. However, the error existing in both escape probabilities is not expected to appear in the final products because the ratio of $P_{e, F}$ and $P_{e, F}^{i s o}$ is used. The error existing in both the numerator and denominator of the shadowing effect correction factor in Eq. 12 can be canceled out.

The collision probabilities from the nonfuel region are expressed as follows:

$$
\begin{gathered}
P_{M i}(u)=\frac{P_{i M}(u) \Sigma_{t, i}(u) V_{i}}{\sum_{p, M} V_{M}} ; \\
P_{M M}(u)=1-\sum_{i \in F} P_{M i}(u)=1-\sum_{i \in F} \frac{P_{i M}(u) \Sigma_{t, i}(u) V_{i}}{\sum_{p, M} V_{M}} .
\end{gathered}
$$

Finally, all the collision probabilities and escape probabilities, which are needed to solve the slowing-down equations in Eqs 4-6, are derived.

\subsection{Calculation Flow}

There are two different options depending on how the collision probabilities of the subdivided regions of the isolated fuel pellet (i.e., $\left.P_{i j}^{i s o}\right)$ are calculated. The flowchart of PSMs is shown in Figure 1. The boxes with the dashed line are processes for only the PSM but not the PSM-CPM. The calculation process is as follows:

1. Read input information in a given problem.

2. Read the 72-group multigroup XS library and the 5-104 equallethargy pointwise energy XS library.

3. Generate the $\hat{P}_{i j}^{i s o}$ vs total XS table for all the pin-geometry types in the given problem by using the CPM solver.

4. Perform the fixed-source MOC transport calculation for the whole-problem domain, and then calculate the individual Dancoff factors of the fuel pins with the enhanced neutron current method (Yamamoto, 2012).

5. Calculate $P_{e, F}^{i s o}$ and $P_{e, F}$ with Carlvik's two-term method (Knott et al., 2010) and then calculate the shadowing effect correction factor $\eta_{F}$ using Eq. 12.

6. Interpolate $\hat{P}_{i j}^{i s}$ from the $\hat{P}_{i j}^{i s o}$ table using the pointwise total XS of the fuel pellet for energy $u$ (PSM) or calculate $P_{i j}^{i s o}$ using the CPM solver with the spatially dependent pointwise total XSs in the fuel pellet for energy $u$ (PSM-CPM).

7. Correct the shadowing effect using the shadowing effect correction factor as in Eq. 13, and then calculate $P_{i j}, P_{i M}$, $P_{M i}$, and $P_{M M}$ with Eqs 13-17.

8. Solve the pointwise energy slowing-down equations in Eqs 4-6. Repeat steps 6-8 for all the pointwise energy points from high to low energy.

9. Condense the pointwise XS to the position-dependent multigroup XS. Consider the resonance upscattering effect for ${ }^{238} \mathrm{U}$ (Choi, 2017; Choi et al., 2017). Return to step 5) until all the fuel pins in the problem are treated.

\section{NUMERICAL RESULTS}

Several LWR problems are solved to verify the accuracy of the PSMs. Table 1 presents a summary of test cases and methods

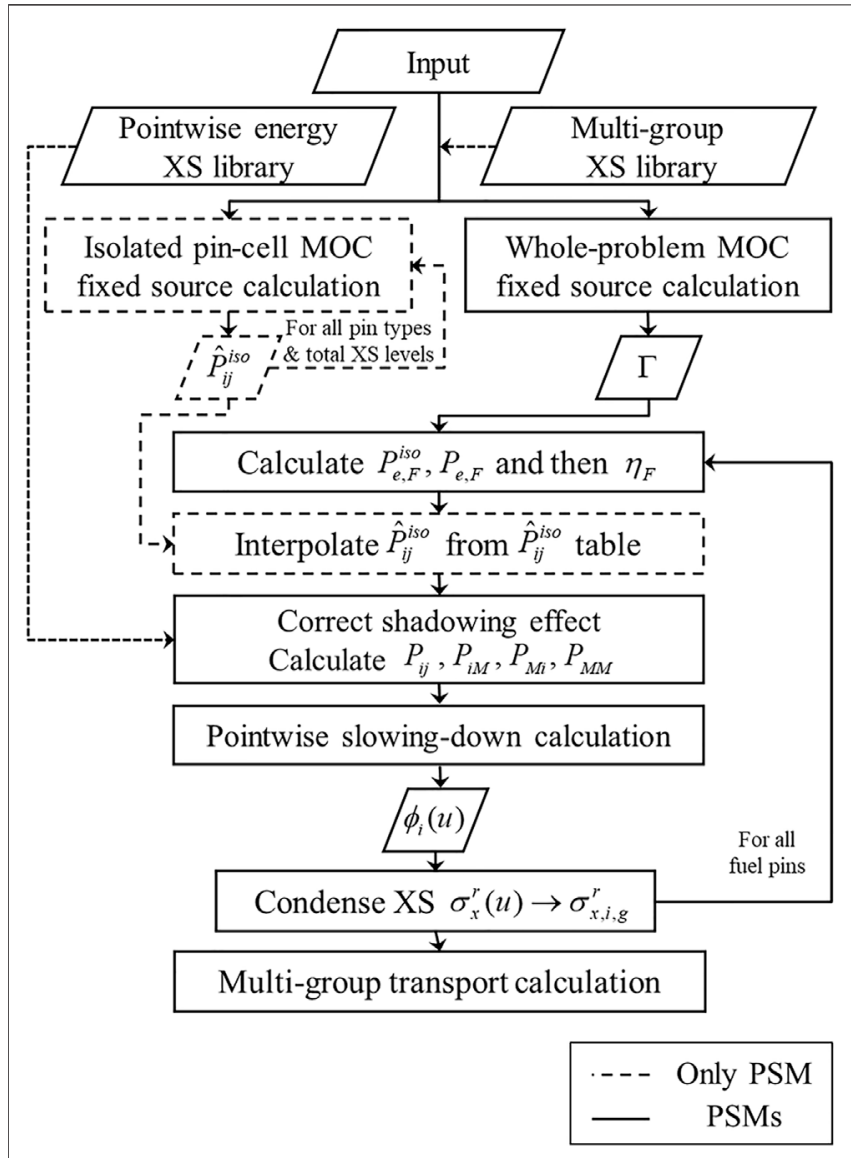

FIGURE 1 | Flowchart of the pin-based pointwise energy slowing-down solution method (PSM) (Choi, 2017; Choi et al., 2017).

used in the verifications. The test cases include the pin-cell and FA geometry with uniform material distribution, uniform temperature profile, and fresh fuel. The methods used in the comparisons are as follows:

1. EQ: The conventional equivalence theory.

2. PSM: The pin-based pointwise slowing-down method with the $\hat{P}_{i j}^{i s o}$ table.

3. PSM-CPM: The pin-based pointwise slowing-down method with the CPM.

The following option was used in all the calculations in Sensitivity Test for Energy Points in the Pointwise Cross-Section Library and VERA $17 \times 17$ Fuel Assembly Problem: MOC condition: $0.01 \mathrm{~cm}$ ray spacing, 128 azimuthal angles, and $\mathrm{T}-\mathrm{Y}$ optimized three polar angles (Yamamoto et al., 2007). It should be also noted that those calculation results in Sensitivity Test for Energy Points in the Pointwise Cross-Section Library and VERA $17 \times 17$ Fuel Assembly Problem were calculated with the $\mathrm{P}_{2}$ high-order scattering model to reduce errors from the anisotropic source. The reference was the MCS Monte Carlo code (Lee et al., 2020). The numerical test was performed with the lattice physics code STREAM (Choi et al., 2015b). STREAM uses the method of 
TABLE 1 | Summary of test cases (Choi, 2017).

\begin{tabular}{|c|c|c|c|c|c|c|}
\hline Section & Test name & Geometry & $\begin{array}{c}\text { Material } \\
\text { distribution }\end{array}$ & $\begin{array}{l}\text { Temperature } \\
\text { profile }\end{array}$ & Method & Note \\
\hline 3.1 & $\begin{array}{l}\text { Sensitivity test for energy points in the PW XS } \\
\text { library }\end{array}$ & Pin-cell & $\begin{array}{l}\text { Uniform, } \\
\text { nonuniform }\end{array}$ & Uniform & PSM, PSM-CPM & $\begin{array}{l}\text { Computing time and } \\
\text { eigenvalue }\end{array}$ \\
\hline 3.2 & VERA FA & FA & Uniform & Uniform & PSM & Various FA types \\
\hline 3.3 & $17 \times 17 \mathrm{FA}$ & FA & Uniform & Uniform & $\begin{array}{l}\text { EQ, PSM, } \\
\text { PSM-CPM }\end{array}$ & Computing time test \\
\hline
\end{tabular}

characteristics (MOC) for the transport calculation and the equivalence theory for resonance treatment.

\subsection{Sensitivity Test for Energy Points in the Pointwise Cross-Section Library}

Sensitivity test was performed to determine a reliable option to give accurate results by PSMs. The parameter to test is the number of energy points in the PW XS libraries.

The number of energy points is important in terms of accuracy and computational efficiency. When many energy points are used, the solution from the slowing-down calculation is accurate. However, the calculation time is proportional to the number of energy points. Four pointwise energy libraries, which have different numbers of energy points, were used in the sensitivity test. A normal $\mathrm{UO}_{2}$ pin-cell depletion problem was solved with PSMs. The reason that the depletion problem was selected is that light nuclides usually do not need many energy points, whereas heavy nuclides need many energy points because of their many resonances in XSs. Pointwise energy XS libraries with $5,000,10,000,50,000$, and 100,000 points were used in the test. The energy between $0.3 \mathrm{eV}$ and $30 \mathrm{keV}$ was divided with equal lethargy depending on the libraries. The result with 100,000 points was set as a reference. From the internal test, it was verified that more than 100,000 points do not have noticeable effect on the results.

Figure 2 shows the results with the four libraries. The result with 50,000 energy points is quite close to the reference. The differences in the eigenvalue are less than $5 \mathrm{pcm}$ over all the depletion steps. The result with 10,000 energy points is also reliable in terms of the eigenvalue. The maximum difference is $17 \mathrm{pcm}$. However, the result with 5,000 energy points is significantly different from the reference, with a maximum difference of $133 \mathrm{pcm}$. From this sensitivity test for the number of energy points, it is verified that 50,000 energy points are sufficient to get an accurate result. In the STREAM code, the pointwise energy XS library with 10,000 points is used as a default. The library is accurate enough to get reasonable solutions for practical use. The library with 50,000 points is used as an option when a user wants to get the most accurate result. All the results in this work were generated with 50,000 energy points to get the highest accuracy. The number of energy points can be further reduced by using a small lethargy width for high energy and a large lethargy width for low energy because resonances at high energy are narrower and more densely distributed.

The calculation times elapsed in PSMs were tested. $\mathrm{A} \mathrm{UO}_{2}$ fuel burnup level of $60 \mathrm{MW} \mathrm{d} / \mathrm{kg}$ was used in this test. The number of

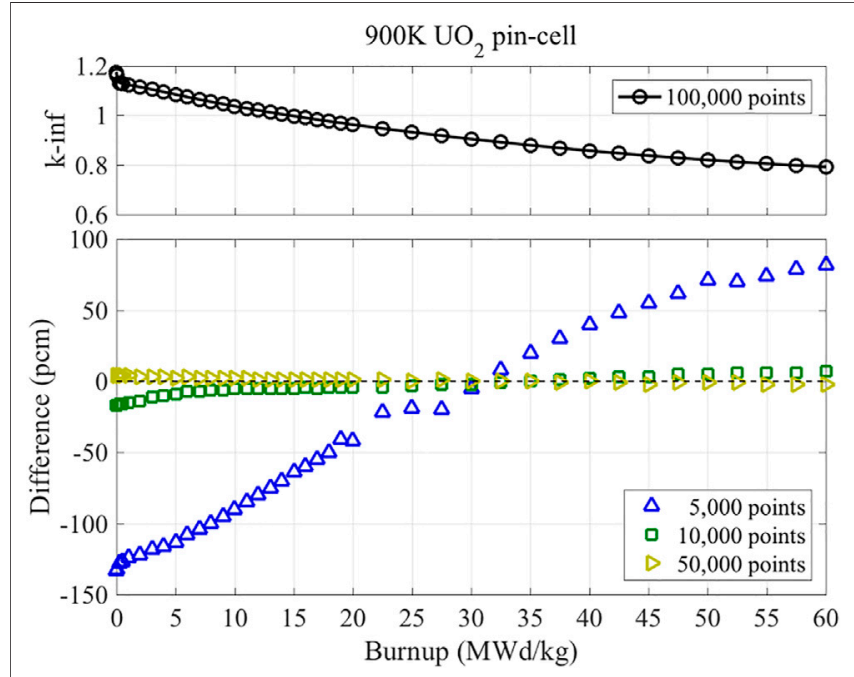

FIGURE 2 | Comparison of k-inf from the PSM with different numbers of energy points in the XS libraries (Choi, 2017).

TABLE 2 | Elapsed time in resonance treatment with PSMs (Choi, 2017).

\begin{tabular}{|c|c|c|c|}
\hline \multicolumn{2}{|l|}{ Method $\rightarrow$} & \multirow{2}{*}{$\frac{\text { PSM-CPM }}{0.79267}$} & \multirow{2}{*}{$\frac{\text { PSM }}{0.79282}$} \\
\hline k-inf $\rightarrow$ & & & \\
\hline \multirow[t]{5}{*}{ Elapsed time (sec) } & PSM solution ${ }^{a}$ & 0.148 & 0.011 \\
\hline & XS condensation ${ }^{\mathrm{b}}$ & 0.102 & 0.115 \\
\hline & Nuclide grouping ${ }^{\mathrm{C}}$ & 0.104 & 0.104 \\
\hline & $\hat{P}_{i j}^{i s o}$ table $^{\mathrm{d}}$ & - & 0.003 \\
\hline & PSM total ${ }^{\mathrm{e}}$ & 0.354 & 0.233 \\
\hline
\end{tabular}

${ }^{a}$ Elapsed time in solving the slowing-down equation and calculating collision probabilities.

${ }^{b}$ Elapsed time in condensing the pointwise XS, to multigroup XS.

${ }^{c}$ Elapsed time in calculating the macroscopic XSs, and the average mass for the each nuclide group.

${ }^{d}$ Elapsed time in generating the collision probability table.

${ }^{\text {e}}$ Total elapsed time in all calculations related to the PSM.

the subdivided regions of the pellet is 5 . Because of the spatial selfshielding effect in the fuel pellet, the material compositions are nonuniform in the pellet. The number of nuclides in the fuel is 198. The library with 50,000 points was used in this test. The eigenvalue results and elapsed time are shown in Table 2.

When the $\hat{P}_{i j}^{i s o}$ table is used to calculate the collision probabilities of the isolated fuel pellet (i.e., $P_{i j}^{i s o}$ ), the elapsed time used in "PSM solution" is reduced by a factor of 13.5. There 


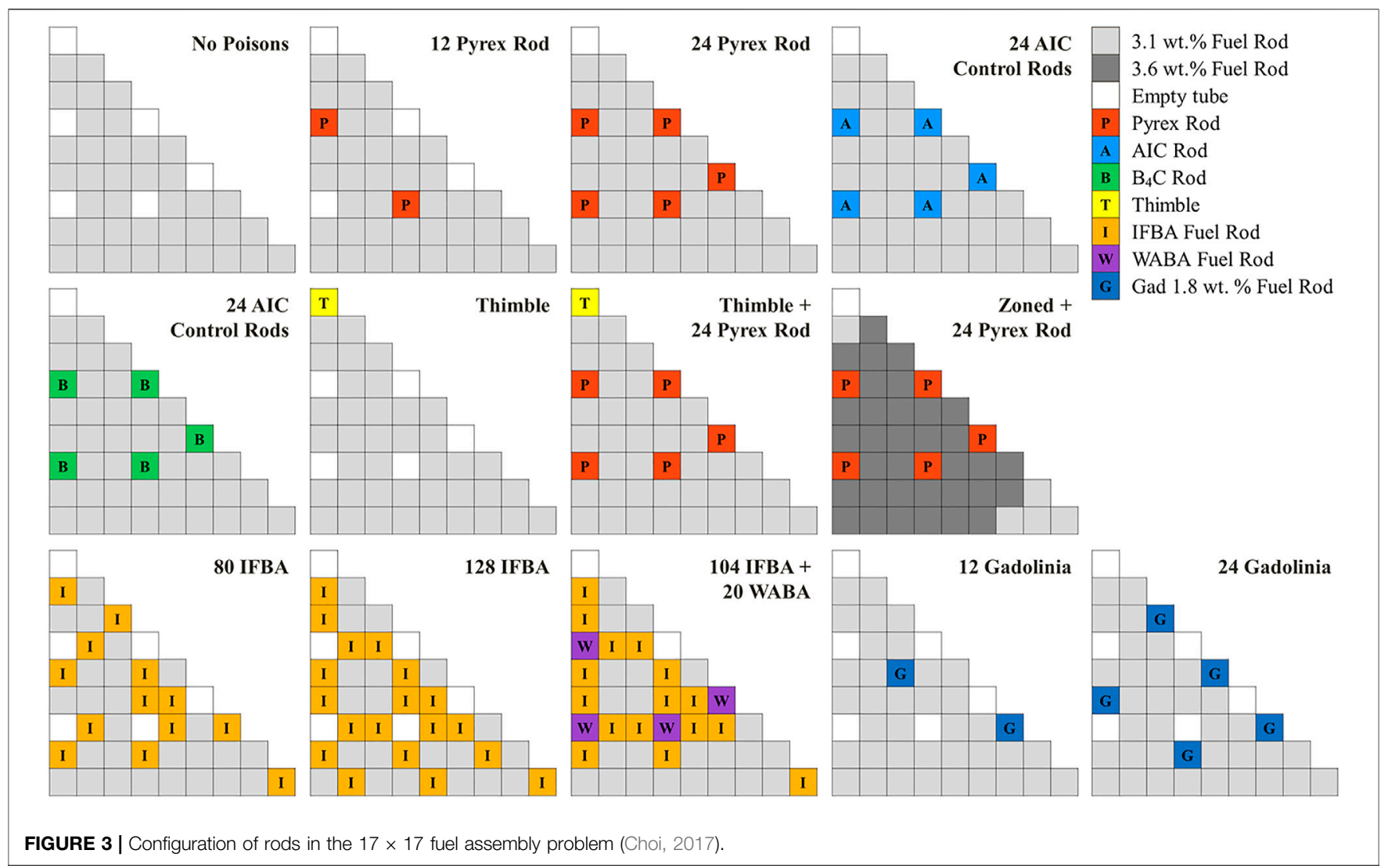

are two error sources for the $\hat{P}_{i j}^{i s o}$ table. One is error from interpolating $\hat{P}_{i j}^{i s o}$. The $\hat{P}_{i j}^{i s o}$ table is generated as a function of the total XS of the fuel pellet. From the internal test, it was concluded that the error from the interpolation is less than 4 $\mathrm{pcm}$. The second error source is in approximation in the $\hat{P}_{i j}^{i s o}$ table. The $\hat{P}_{i j}^{i s o}$ table is made with a constant pointwise XS approximation in the fuel pellet. Overall, an error of $15 \mathrm{pcm}$ occurs from the $\hat{P}_{i j}^{i s o}$ table. The second error source is the major difference between PSM and PSM-CPM. More detailed comparisons are performed in the accompanying article (Kim et al., 2021). The $\hat{P}_{i j}^{i s o}$ table effectively reduces the calculation time without a significant loss of accuracy. The additional calculation time required in generating the $\hat{P}_{i j}^{i s o}$ table is $0.003 \mathrm{~s}$, which is negligible. The PSM takes $0.233 \mathrm{~s}$ to calculate the effective multigroup XS of the burned fuel pellet with five submeshes. Although the XS condensation and the grouping are quite a simple calculation, they are the major time-consuming tasks in PSMs. The code must be further optimized to reduce the calculation time in the XS condensation and the grouping. With the 10,000-point library, the calculation time can be further reduced by a factor of 5 .

\subsection{VERA $17 \times 17$ Fuel Assembly Problem}

$17 \times 17$ fuel assembly (FA) problems were solved to verify PSMs. The $17 \times 17$ FAs in the VERA benchmark problem (Godfrey, 2014) were selected and solved as described in this section. Figure 3 shows the configuration of the rods for various types of FAs. Various burnable poisons and control rods were used in the FA design. Table 3 summarizes the material information and short descriptions of the FAs. The detailed specifications of the geometry and the material compositions are well described in the reference (Godfrey, 2014). The solutions for the $17 \times 17$ FAs were generated by the PSM. The fuel pellets used in this problem have a uniform material composition and a uniform temperature profile.

The results for $\mathrm{k}$-inf and the pin power distribution were obtained with PSMs as shown in Table 4 and Table 5, respectively. PSMs show quite accurate and consistent results. The differences in k-inf are of the order of $100 \mathrm{pcm}$. For the FA with AIC control rods, the PSM-CPM has a difference of $216 \mathrm{pcm}$ in $\mathrm{k}$-inf. The RMS difference and the maximum difference in the power distribution are approximately 0.17 and $0.41 \%$, respectively.

From the verification with the various types of $17 \times 17$ FAs, it is verified that PSMs calculate accurate and consistent results in $\mathrm{k}$-inf and the pin power distribution.

The PSM and PSM-CPM calculate identical solutions for the condition (i.e., a uniform material composition and temperature profile in the fuel pellet). There is a slight difference (i.e., less than $3 \mathrm{pcm}$ in the eigenvalue) between PSM and PSM-CPM caused by the $\hat{P}_{i j}^{i s o}$ table interpolation.

\subsection{Test for Computing Time}

The PSM and PSM-CPM showed high accuracy in the reactor parameters for the various verification problems. In order to use 
TABLE 3 | Description for the fuel assembly problem (Choi, 2017).

\begin{tabular}{|c|c|c|c|c|c|c|}
\hline Problem & Description & $\begin{array}{c}\mathrm{UO}_{2} \text { enrichment } \\
(\%)\end{array}$ & $\begin{array}{l}\text { Moderator temperature } \\
\text { (K) }\end{array}$ & $\begin{array}{l}\text { Fuel temperature } \\
\text { (K) }\end{array}$ & $\begin{array}{c}\text { Moderator density } \\
\text { (g/cc) }\end{array}$ & $\begin{array}{c}\text { Boron concentration } \\
\text { (ppm) }\end{array}$ \\
\hline A & No poison & 3.1 & 565 & 565 & 0.743 & 1,300 \\
\hline B & No poison & & 600 & 600 & 0.661 & \\
\hline C & No poison & & & 900 & & \\
\hline D & No poison & & & 1,200 & & \\
\hline$E$ & 12 Pyrex & & & 600 & 0.743 & \\
\hline $\mathrm{F}$ & 24 Pyrex & & & & & \\
\hline 1 & Thimble & & & & & \\
\hline J & Thimble, 24 Pyrex & & & & & \\
\hline $\mathrm{K}$ & Zoned, 24 Pyrex & $3.1,3.6$ & & & & \\
\hline $\mathrm{L}$ & 80 IFBA & 3.1 & & & & \\
\hline M & 128 IFBA & & & & & \\
\hline $\mathrm{N}$ & 104 IFBA, 20 WABA & & & & & \\
\hline
\end{tabular}

TABLE 4 | k-inf results-PSM and PSM-CPM.

\begin{tabular}{|c|c|c|c|c|c|c|}
\hline \multirow[t]{2}{*}{ Problem } & \multirow[t]{2}{*}{ Description } & \multicolumn{5}{|c|}{$k$-inf } \\
\hline & & MCS & PSM & Difference (pcm) & PSM-CPM & Difference $(\mathrm{pcm})$ \\
\hline$A$ & No poison & $1.18165 \pm 0.00007$ & 1.18179 & 14 & 1.18181 & 16 \\
\hline C & No poison & $1.17371 \pm 0.00008$ & 1.17357 & -14 & 1.17360 & -11 \\
\hline D & No poison & $1.16597 \pm 0.00007$ & 1.16567 & -30 & 1.16570 & -27 \\
\hline$E$ & 12 Pyrex & $1.06915 \pm 0.00007$ & 1.06922 & 7 & 1.06924 & 9 \\
\hline $\bar{F}$ & 24 Pyrex & $0.97554 \pm 0.00007$ & 0.97569 & 15 & 0.97571 & 17 \\
\hline । & Thimble & $1.17931 \pm 0.00007$ & 1.17960 & 29 & 1.17962 & 31 \\
\hline J & Thimble, 24 Pyrex & $0.97475 \pm 0.00007$ & 0.97494 & 19 & 0.97497 & 22 \\
\hline K & Zoned, 24 Pyrex & $1.01944 \pm 0.00007$ & 1.01987 & 43 & 1.01989 & 45 \\
\hline L & 80 IFBA & $1.01837 \pm 0.00007$ & 1.01896 & 59 & 1.01898 & 61 \\
\hline M & 128 IFBA & $0.93838 \pm 0.00007$ & 0.93914 & 76 & 0.93916 & 78 \\
\hline N & 104 IFBA, 20 WABA & $0.86919 \pm 0.00007$ & 0.86977 & 58 & 0.86978 & 59 \\
\hline
\end{tabular}

the PSM and PSM-CPM in a practical design, it should be confirmed that they calculate the effective multigroup XS within a reasonable computation time.

The $17 \times 17$ FA problem was selected for the computation time comparison. The EQ was compared to the PSM. The FA was modeled with octant symmetry. For this section, the following option was used in the calculation: MOC condition: $0.05 \mathrm{~cm}$ ray spacing, 48 azimuthal angles, and three polar angles. In the test, each pin-cell had eight azimuthal sectors, three radial subregions in the coolant, and five radial subregions in the fuel pellet. The number of flat source regions is 2,842 . The number of macroscopic XS sets is 242. The inflow transport corrected P0 (TCP0) model is used for both options (Choi et al., 2015c). Generally, these MOC conditions and the TCP0 model are used for practical calculations.

The time comparison results are shown in Table 6. The results were generated on an OSX system with a 3.1-GHz Intel Core i7 processor. PSMs perform the energy-independent fixed-source calculations to consider the shadowing effect. However, the EQ needs 15 fixed-source solutions for the fuel. The STREAM code performs the fixed-source MOC calculation for the resonance energy groups above $4 \mathrm{eV}$ (Choi et al., 2015a). In case of the $17 \times$ 17 FA problem, the Dancoff factors are calculated for the fuel and the cladding. PSMs are not applied on the resonance treatment for the cladding. Both PSMs and EQ use a common resonance treatment method (Choi et al., 2015a) for the cladding. The cladding resonance treatment method is based on the equivalence theory, and therefore PSMs and EQ perform energy-group-dependent MOC fixed-source calculations. Finally, PSM requires 16 MOC fixed-source solutions ( 1 for the fuel; 15 for the cladding), whereas EQ requires 30 fixed-source solutions (15 for the fuel; 15 for the cladding). This is why PSMs need approximately half the computation time in the fixed-source calculation (MOC FSP in Table 6) compared to EQ. Obviously, the elapsed time in the fixed-source MOC calculation depends on the MOC ray conditions. PSMs solve the slowing-down equations for the individual fuel pins. In the case of the $17 \times 17 \mathrm{FA}$ problem with 
TABLE 5 | Pin power distribution results-PSM and PSM-CPM.

\begin{tabular}{|c|c|c|c|c|c|}
\hline \multirow[t]{3}{*}{ Problem } & \multirow[t]{3}{*}{ Description } & \multicolumn{4}{|c|}{ Pin power difference (\%) } \\
\hline & & \multicolumn{2}{|c|}{ PSM vs MCS } & \multicolumn{2}{|c|}{ PSM-CPM vs MCS } \\
\hline & & RMS & Max & RMS & Max \\
\hline$A$ & No poison & $0.116 \pm 0.001$ & $0.217 \pm 0.001$ & $0.116 \pm 0.001$ & $0.217 \pm 0.001$ \\
\hline $\mathrm{B}$ & No poison & $0.103 \pm 0.001$ & $0.205 \pm 0.001$ & $0.103 \pm 0.001$ & $0.205 \pm 0.001$ \\
\hline C & No poison & $0.151 \pm 0.001$ & $0.345 \pm 0.001$ & $0.151 \pm 0.001$ & $0.345 \pm 0.001$ \\
\hline $\mathrm{D}$ & No poison & $0.127 \pm 0.001$ & $0.254 \pm 0.001$ & $0.127 \pm 0.001$ & $0.254 \pm 0.001$ \\
\hline$E$ & 12 Pyrex & $0.109 \pm 0.001$ & $0.303 \pm 0.001$ & $0.109 \pm 0.001$ & $0.303 \pm 0.001$ \\
\hline $\mathrm{F}$ & 24 Pyrex & $0.126 \pm 0.001$ & $0.264 \pm 0.001$ & $0.126 \pm 0.001$ & $0.264 \pm 0.001$ \\
\hline$G$ & $24 \mathrm{AIC}$ & $0.172 \pm 0.001$ & $0.357 \pm 0.002$ & $0.172 \pm 0.001$ & $0.357 \pm 0.001$ \\
\hline $\mathrm{H}$ & 24 B4C & $0.153 \pm 0.001$ & $0.408 \pm 0.002$ & $0.153 \pm 0.001$ & $0.408 \pm 0.001$ \\
\hline 1 & Thimble & $0.143 \pm 0.001$ & $0.340 \pm 0.001$ & $0.143 \pm 0.001$ & $0.340 \pm 0.001$ \\
\hline$J$ & Thimble, 24 Pyrex & $0.153 \pm 0.001$ & $0.351 \pm 0.001$ & $0.153 \pm 0.001$ & $0.351 \pm 0.001$ \\
\hline K & Zoned, 24 Pyrex & $0.129 \pm 0.001$ & $0.297 \pm 0.002$ & $0.129 \pm 0.001$ & $0.297 \pm 0.002$ \\
\hline$L$ & 80 IFBA & $0.108 \pm 0.001$ & $0.317 \pm 0.001$ & $0.108 \pm 0.001$ & $0.317 \pm 0.001$ \\
\hline$M$ & 128 IFBA & $0.140 \pm 0.001$ & $0.285 \pm 0.002$ & $0.140 \pm 0.001$ & $0.285 \pm 0.002$ \\
\hline$N$ & 104 IFBA, 20 WABA & $0.154 \pm 0.001$ & $0.414 \pm 0.002$ & $0.154 \pm 0.001$ & $0.414 \pm 0.002$ \\
\hline O & 12 Gadolinia & $0.148 \pm 0.001$ & $0.370 \pm 0.001$ & $0.148 \pm 0.001$ & $0.370 \pm 0.001$ \\
\hline$P$ & 24 Gadolinia & $0.159 \pm 0.001$ & $0.339 \pm 0.001$ & $0.159 \pm 0.001$ & $0.339 \pm 0.001$ \\
\hline
\end{tabular}

TABLE 6 | Comparison for elapsed time (unit: s) (Choi, 2017).

\begin{tabular}{lccc} 
Category & EQ & PSM & PSM-CPM \\
\hline Reading library $^{\mathrm{a}}$ & 0.36 & 0.37 & 0.37 \\
MOC FSP solver for fuel $^{\mathrm{b}}$ & 0.36 & 0.03 & 0.03 \\
MOC FSP solver for cladding $^{\mathrm{C}}$ & 0.36 & 0.35 & 0.36 \\
Interpolation in multigroup XS and RI libraries $^{\mathrm{d}}$ & 0.97 & 0.15 & 0.14 \\
Nuclide grouping $^{\mathrm{e}}$ & - & 0.23 & 0.22 \\
XS condensation $^{\mathrm{f}}$ & & & \\
Slowing-down solver $^{\mathrm{g}}$ & - & 0.42 & 5.21 \\
Total XS generation $^{\mathrm{h}}$ & 2.25 & 1.67 & 6.44 \\
Total simulation & 7.78 & 7.16 & 11.95 \\
\hline
\end{tabular}

${ }^{a}$ Elapsed time in reading the $X S$ and $R I$ libraries.

${ }^{b}$ Elapsed time in solving the MOC fixed-source problem for the fuel.

${ }^{c}$ Elapsed time in solving the MOC fixed-source problem for the cladding.

${ }^{d}$ Elapsed time in interpolating the multigroup XS and RI, from the multigroup XS library and the Rl library.

${ }^{e}$ Elapsed time in calculating the macroscopic pointwise energy XSs of the nuclide groups.

${ }^{t}$ Elapsed time in collapsing the pointwise energy XS to the multigroup XSs.

${ }^{g}$ Elapsed time in solving the slowing-down equation and calculating the collision probabilities.

${ }^{n}$ Total elapsed time in calculating the multigroup XSs.

octant symmetry, the slowing-down equations are solved for 39 fuel pins. Approximately $0.41 \mathrm{~s}$ is spent on the pointwise energy slowing-down calculations for all the fuel pins in the problem. PSMs spend additional time on grouping the nuclides and the energy condensations. A non-negligible time is consumed for these calculations, although the calculations are quite simple. Because the pointwise energy XS data are used in PSMs, these calculations are inevitable. The PSM-CPM takes a longer time in the slowing-down solver because it calculates the collision probability with the CPM solver for all energy points. PSMs spend less time in interpolating the RI from the multigroup RI library because they do not use the RI look-up table to calculate the effective XSs of fuel materials.

The same problem was solved with different numbers of the radially subdivided regions in the fuel pellet. Figure 4 shows the

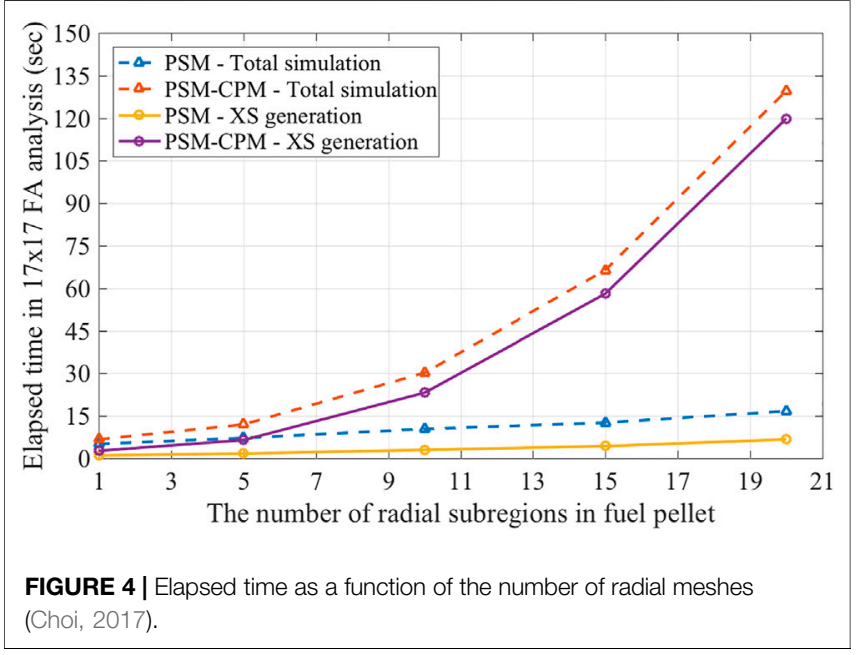

calculation time as a function of the number of the subdivided regions in the fuel pellet. Both PSM and PSM-CPM were tested with different numbers of regions. When the number of radially subdivided regions is small, the differences in the calculation time between PSMs are not noticeable. As the number of the subdivided regions increases, the elapsed time used in the XS generation significantly increases with the PSM-CPM. With the PSM-CPM, the XS generation accounts for a very large portion of the total simulation. However, the elapsed time in the XS generation with the PSM is not very long compared to the total simulation time. The PSM is very effective in reducing the calculation time in the XS generation.

In conclusion, PSMs can calculate the multigroup XS within a reasonable computation time. PSMs save the calculation time by reducing the number of MOC fixed-source calculations. Even though PSMs solve the pointwise energy slowing-down equations, the calculation time is not problematic because various techniques are applied to enhance the performance of PSMs (Choi, 2017). The PSM-CPM takes a longer time in 
calculating the multigroup XSs with many subdivided regions in the fuel pellet. Nevertheless, the PSM-CPM is still useful because more than five subdivided regions are hardly used in the practical calculations for $\mathrm{UO}_{2}$ pin-cells. For a uniform material distribution and temperature profile, PSMs show consistent results. The comparative analysis between PSM and PSM-CPM for a nonuniform material distribution and temperature profile is presented in the accompanying article (Kim et al., 2021).

\section{CONCLUSION}

The PSM has been refined to exactly consider the collision probability in the subdivided regions of the isolated fuel pellet. The collision probabilities of an isolated pellet with the radial subdivisions are calculated by using the CPM. The PSM calculates the collision probability corresponding to the grid of the total XSs which is assumed to be constant in all the subdivided regions of the fuel pellet before solving the slowing-down equation. Then, the PSM uses a pre-generated look-up table for the collision probability to reduce the calculation time, but it is only valid if the fuel pellet has a uniform material composition and temperature profile in the subdivided regions of the fuel pellet. On the other hand, the PSM-CPM directly calculates the collision probability in the fuel pellet while solving the slowing-down equation so that exact collision probabilities in all the subdivided regions for the isolated fuel pellet are calculated.

The PSM-CPM has been verified with a few types of LWR FA. PSMs generate consistent results for specified problems in this article. The verification calculations showed good agreement in the eigenvalues, with differences of the order of $100 \mathrm{pcm}$ compared to those of the reference solutions. The pin power distributions were

\section{REFERENCES}

Carlvik, I. (1964). A Method for Calculating Collision Probabilities in General Cylindrical Geometry and Applications to Flux Distributions and Dancoff Factors. Stockholm (Sweden): Aktiebolaget Atomenergi. A/CONF. 28/P/681.

Choi, S., Khassenov, A., and Lee, D. (2015). Resonance Self-Shielding Method Using Resonance Interference Factor Library for Practical Lattice Physics Computations of LWRs. J. Nucl. Sci. Technol. 53, 1142-1154. doi:10.1080/ 00223131.2015 .1095686

Choi, S., Lee, H., Hong, S. G., and Lee, D. (2015). Resonance Self-Shielding Methodology of New Neutron Transport Code Stream. J. Nucl. Sci. Technol. 52, 1133-1150. doi:10.1080/00223131.2014.993738

Choi, S., Smith, K., Lee, H. C., and Lee, D. (2015). Impact of Inflow Transport Approximation on Light Water Reactor Analysis. J. Comput. Phys. 299, 352-373. doi:10.1016/j.jcp.2015.07.005

Choi, S., Lee, C., and Lee, D. (2017). Resonance Treatment Using Pin-Based Pointwise Energy Slowing-Down Method. J. Comput. Phys. 330, 134-155. doi:10.1016/j.jcp.2016.11.007

Choi, S. (2017). Pin-Based Pointwise Energy Slowing-Down Method for Resonance Self-Shielding Calculation. Doctoral Thesis. Ulsan National Institute of Science and Technology.

Godfrey, A. T. (2014). VERA Core Physics Benchmark Progression Problem Specifications. Oak Ridge National Laboratory, Oak Ridge National Laboratory. CASL-U-2012-0131-004. also sufficiently accurate. It has also been demonstrated that the computation times using the PSM-CPM are comparable to those with the conventional equivalence theory methods in the practical use. The accompanying article demonstrates more comparative analysis to verify the PSM-CPM for nonuniform material and temperature distributions in the fuel pellet.

\section{DATA AVAILABILITY STATEMENT}

The original contributions presented in the study are included in the article/Supplementary Material; further inquiries can be directed to the corresponding author.

\section{AUTHOR CONTRIBUTIONS}

SC contributed to the conception and design of the methodology. SC wrote the first draft of the manuscript. SC and WK wrote sections of the manuscript. WK reviewed and edited the manuscript. DL supervised all the activity for the manuscript. All authors contributed to manuscript revision and read and approved the submitted version.

\section{FUNDING}

This work was supported by the National Research Foundation of Korea (NRF) grant funded by the government of Korea (MSIT) (No. NRF-2019M2D2A1A03058371). This work was partially supported by Korea Institute of Energy Technology Evaluation and Planning (KETEP) grant funded by the Korea government (MOTIE) (20206510100040).

Kim, W., Choi, S., and Lee, D. (2021). Refinements of Pin-Based Pointwise Energy Slowing-Down Method for Resonance Self-Shielding Calculation-II: Verifications. Front. Energy Res. 9, 765865. doi:10.3389/fenrg.2021.765865

Knott, D., and Yamamoto, A. (2010). "Lattice Physics Computations," in Handbook of Nuclear Engineering. Editor D.G. Cacuci (Boston, MA: Springer US), 913-1239. doi:10.1007/978-0-387-98149-9_9

Koike, H., Yamaji, K., Kirimura, K., Sato, D., Matsumoto, H., and Yamamoto, A. (2012). Advanced Resonance Self-Shielding Method for gray Resonance Treatment in Lattice Physics Code GALAXY. J. Nucl. Sci. Technol. 49, 725-747. doi:10.1080/00223131.2012.693885

Lee, H., Kim, W., Zhang, P., Lemaire, M., Khassenov, A., Yu, J., et al. (2020). MCS - A Monte Carlo Particle Transport Code for Large-Scale Power Reactor Analysis. Ann. Nucl. Energ. 139, 107276. doi:10.1016/ j.anucene.2019.107276

Powney, D. J., and Hutton, J. L. (2002). The Next Generation WIMS Lattice Code : WIMS9. Seoul, Korea: PHYSOR-2002.

Rhodes, J., Smith, K., and Lee, D. (2006). CASMO-5 Development and Applications. Vancouver, BC, Canada: PHYSOR-2006.

Stamm'ler, R. J. J., and Abbate, M. J. (1983). Methods of Steady-State Reactor Physics in Nuclear Design. London: Academic Press.

Stoker, C. C., and Weiss, Z. J. (1996). Spatially Dependent Resonance Cross Sections in a Fuel Rod. Ann. Nucl. Energ. 23, 765-778. doi:10.1016/03064549(95)00074-7

Yamamoto, A., Tabuchi, M., Sugimura, N., Ushio, T., and Mori, M. (2007). Derivation of Optimum Polar Angle Quadrature Set for the Method of 
Characteristics Based on Approximation Error for the Bickley Function. J. Nucl. Sci. Technol. 44, 129-136. doi:10.1080/18811248.2007.9711266

Yamamoto, A. (2012). Evaluation of Background Cross Section for Heterogeneous and Complicated Geometry by the Enhanced Neutron Current Method. J. Nucl. Sci. Technol. 45, 1287-1292. doi:10.1080/ 18811248.2008.9711916

Zhang, Q., Jiang, R., Zhao, Q., Cao, L., and Wu, H. (2018). Accurate Resonance Absorption Calculation for Fuel Pins with Non-uniform Intra-pellet Temperature Profile Based on ultra-fine-group SlowingDown Calculations. Ann. Nucl. Energ. 120, 392-401. doi:10.1016/ j.anucene.2018.06.005

Zhang, Q., Shuai, Q., Zhao, Q., Liang, L., Wu, H., and Cao, L. (2020). Improvements on the Method of Ultra-Fine-Group SlowingDown Solution Coupled with Method of Characteristics on Irregular Geometries. Ann. Nucl. Energ. 136, 1070171. doi:10.1016/ j.anucene.2019.107017
Conflict of Interest: The authors declare that the research was conducted in the absence of any commercial or financial relationships that could be construed as a potential conflict of interest.

Publisher's Note: All claims expressed in this article are solely those of the authors and do not necessarily represent those of their affiliated organizations, or those of the publisher, the editors, and the reviewers. Any product that may be evaluated in this article, or claim that may be made by its manufacturer, is not guaranteed or endorsed by the publisher.

Copyright $\odot 2021$ Choi, Kim and Lee. This is an open-access article distributed under the terms of the Creative Commons Attribution License (CC BY). The use, distribution or reproduction in other forums is permitted, provided the original author(s) and the copyright owner(s) are credited and that the original publication in this journal is cited, in accordance with accepted academic practice. No use, distribution or reproduction is permitted which does not comply with these terms. 